Relations industrielles

Industrial Relations

\title{
Relations industrielles : Acteurs, auteurs, faits, tendances, par Dimitri Weiss, (Collection administrative des Entreprises), Paris, Éditions Sirey, 1973, 320 pp.
}

\section{Félix Quinet}

Volume 28, numéro 3, 1973

URI : https://id.erudit.org/iderudit/028427ar

DOI : https://doi.org/10.7202/028427ar

Aller au sommaire du numéro

Éditeur(s)

Département des relations industrielles de l'Université Laval

ISSN

0034-379X (imprimé)

1703-8138 (numérique)

Découvrir la revue

Citer ce compte rendu

Quinet, F. (1973). Compte rendu de [Relations industrielles : Acteurs, auteurs, faits, tendances, par Dimitri Weiss, (Collection administrative des Entreprises), Paris, Éditions Sirey, 1973, 320 pp.] Relations industrielles / Industrial Relations, 28(3), 664-665. https://doi.org/10.7202/028427ar

Tous droits réservés @ C Département des relations industrielles de l'Université Laval, 1973
Ce document est protégé par la loi sur le droit d'auteur. L'utilisation des services d’Érudit (y compris la reproduction) est assujettie à sa politique d'utilisation que vous pouvez consulter en ligne.

https://apropos.erudit.org/fr/usagers/politique-dutilisation/ 


\section{RECENSIONS}

Relations industrielles : Acteurs, auteurs, faits, tendances, par Dimitri Weiss, (Collection Administration des Entreprises), Paris, Editions Sirey, 1973, 320 pp.

Le livre récent que Dimitri Weiss qui dirige l'enseignement des relations industrielles à l'Institut d'Administration des Entreprises et à l'U.E.R. Gestion de l'Université de Paris 1 (Panthéon - Sorbonne) - a consacré aux relations industrielles est un ouvrage à lire dans sa totalité par tous les spécialistes de cette discipline et même par tous ceux qu'elle intéresse. Un grand nombre de livres qui portent sur les problèmes du Travail et qui sont écrits par des spécialistes européens sont très souvent - et pour des raisons qu'il ne nous appartient pas de juger - fortement teintés de préoccupations idéologiques. $\mathrm{Ce}$ n'est pas le cas du livre écrit par Dimitri Weiss. L'auteur nous a semblé fondamentalement préoccupé par l'importance grandissante de la fonction «relations industrielles», en tant qu'ensemble de données et de variables qui mérite son autonomie et, par conséquent, a droit à des études et à des analyses qui lui soient totalement consacrées. Dans le cadre de cette préoccupation que je crois pouvoir lui attribuer, Dimitri Weiss on lui laissera évidemment la responsabilité de ses vues et de ses choix - recueille systématiquement, en les intégrant fort utilement, les interprétations, analyses et réflexions de maints syndicalistes, employeurs, sociologues, universitaires parmi lesquels on compte nombre de Nord-Américains - concernant les multiples problèmes qui se posent aujourd'hui dans le domaine des relations industrielles, en France et ailleurs. Citons quelques-uns des problèmes traités dans l'ouvrage; problèmes d'évaluation: l'étude «behavioriste» des relations industrielles; droit du travail et pouvoir dans l'entreprise; sciences du comportement et direction du personnel; la réalité conflictuelle; problèmes d'incidence pratique : «élargissement» ou «enrichissement » des tâches; l'environnement physique; l'horaire libre; les revendications qualitatives. En faisant le large tour d'horizon du champ des relations industrielles tel qu'il se présente aujourd'hui, l'auteur a non seulement dressé un inventaire substantiel et utile de ses «acteurs, auteurs, faits et tendances $»$, il a aussi procédé à une non moins utile mise en ordre. A ce propos, il faut souligner le soin particulier que l'auteur apporte à définir et à cerner certains concepts de base en relations industrielles, à commencer par le concept de relations industrielles lui-même. Il faut souligner aussi les distinctions qu'il fait entre, par exemple, «relations de travail» et «relations dans le travail » dans l'entreprise, etc. De telles distinctions, si elles n'ont pas valeur de finalité, ne peuvent que faciliter des discussions et des études dont le cadre, grâce à ces distinctions, aura été, au préalable, mieux fixé.

Devant le large éventail des questions soulevées et examinées dans l'ouvrage, il sera difficile au lecteur, probablement en raison de ses propres intérêts et recherches, de ne pas avoir certaines préférences pour telle ou telle question qui a été traitée. Certains lecteurs prendront connaissance avec intérêt de l'analyse que l'auteur a présentée des accordspilote à la Régie Renault (1970), et de la Convention sociale E.G.F. (Electricité et Gaz de France) (1969). D'autres lecteurs trouveront particulièrement intéressant le chapitre consacré à «La Négociation collective de branche, interprofessionnelle et 《au sommet», 》 chapitre où il est dit, entre autres choses, que la loi française du 13 juillet 1971 "permet aux accords d'entreprise d'élaborer un véritable statut collectif du personnel, à défaut de convention collective au plan professionnel. Mais lorsque cette dernière existe - nationale, régionale ou locale - le domaine de l'accord d'entreprise se limite à adapter les dispositions de la convention professionnelle aux conditions particulières de l'entreprise considérée, pouvant fixer en outre, le taux des salaires effectifs..., et celui des accessoires de salaire, et comporter des dispositions nouvelles et des clauses plus favorables aux travailleurs... Dans le cas où une convention collective nationale, régionale ou locale 
viendrait à s'appliquer à l'entreprise postérieurement à la conclusion de la convention d'entreprise, cette dernière devra adapter ses dispositions moins favorables à celles de la convention nouvellement signée ou étendue par arrêté ministériel » (p. 207). D'autres lecteurs enfin, porteront leur attention sur ces sections du livre où l'auteur discute de l'impact que l'intégration européenne pourrait avoir sur les structures et modes d'action des syndicats européens. Parallèlement, la section du livre que l'auteur consacre à la «société anonyme enropéenne 》 pourrait intéresser vivement le spécialiste en relations industrielles, qu'il oeuvre de ce côté-ci, ou de l'autre, de l'Atlantique.

Mais tout est à lire dans l'ouvrage de Dimitri Weiss. Tout en laissant à l'auteur la responsabilité de ses thèses, de ses jugements, et de ses orientations, il faut reconnaître que ce remarquable et dense ouvrage, écrit dans une langue concise et claire, et donc de lecture agréable et captivante, réussit - tout en accordant une attention prioritaire aux problèmes tels qu'ils se posent en France, ce qui est normal puisque l'auteur est français - à faire passer sur le thème des «relations industrielles» le souffle serein d'une perspective internationale qui se veut avant tout fonctionnelle. Le livre de Dimitri Weiss mérite de recevoir bon accueil au Canada: dans nos Universités et nos institutions d'enseignement où il constituera un outil de travail et de référence précieux; dans notre monde des 《relations industrielles », où il sera une source d'évaluations et d'informations bien intégrées et parfaitement actuelles.

Félix QUINET

Commission des relations de travail dans la fonction publique Ottawa

Le monde ouvrier au Québec, bibliographie rétrospective par André $\mathrm{E}$. LeBlanc et James D. Thwaites, Montréal, Les Presses de l'université du Québec, 1973, 283 pp.

Il y a quelques années la direction de Relations industrielles recevait pour publication une bibliographie sur les travailleurs au Québec. Grandement intéressé par le sujet, nous avons communiqué à l'auteur, André LeBlanc, nos commentaires avec des suggestions. Celui-ci a repris son texte et nous a présenté une nouvelle version grandement enrichie. C'est alors que nous avons mis l'auteur en contact avec un autre chercheur du département d'histoire de l'université Laval, James D. Thwaites, qui travaillait sur le même sujet. Le résultat de leur collaboration a pris une ampleur telle qu'il dépassait les cadres d'un article pouvant être publié dans Relations industrielles et fait aujourd'hui l'objet de l'ouvrage Le monde ouvrier au Québec bibliographie rétrospective édité par les Presses de l'université du Québec.

Après avoir suivi de près l'élaboration de cette recherche, nous ne pouvons que nous réjouir de sa publication. Ce n'est pas le premier ouvrage du genre. Sans être encore exhaustif, il nous apparaît le plus complet. Les auteurs ont relevé près de 2,500 titres qui ont été classés selon un ordre thématique comprenant huit divisions : le mouvement ouvrier; les syndicats et métiers; les conflits de travail; le travail; le milieu social; les réponses et réactions; la presse ouvrière ; bibliographies. Un excellent index analytique des auteurs et des groupements facilite la consultation.

Un ouvrage du genre comporte fatalement une bonne part d'arbitraire tant dans la délimitation du sujet que dans le choix des titres. En effet, il n est pas toujours facile de déterminer une frontière précise entre les études qui portent strictement sur le monde ouvrier au Québec et celles qui englobent tout le monde ouvrier au Canada dont celui du Québec. Il en va de même à propos de celles qui, tout en traitant un sujet plus vaste, apportent des éclairages importants sur le monde des travailleurs.

Une recherche du genre n'étant jamais terminée, il faut savoir gré aux auteurs de n'avoir pas attendu le chef d'oeuvre avant d'offrir aux chercheurs un instrument utile qui contribuera sûrement à faciliter leur travail.

\section{Université Laval}

Gérard DION

Collective Bargaining in the Public Service, Toronto, Ontario, The Institute of Public Administration of Canada, 1973, $105 \mathrm{pp}$.

Ce livre, dont la version française paraîtra prochainement, est le compte rendu d'un séminaire sur la négociation collective dans le secteur public qui avait 